\title{
TECHNIQUES AND RESULTS OF DIRECT-ACCESS MINIMALLY INVASIVE MITRAL VALVE SURGERY: A PARADIGM FOR THE FUTURE
}

Lishan Aklog, MD

David H. Adams, MD

Gregory S. Couper, MD

Reuben Gobezie, BA

Samuel Sears, BA

Lawrence H. Cohn, MD
Objectives: Our objective was to determine whether direct-access minimally invasive mitral valve surgery can improve recovery and cost while maintaining the efficacy of conventional surgery. Methods: Minimally invasive mitral valve operations were performed on 106 patients, $58 \%$ male, average age 58.1 years, with good ventricular function. Ninety underwent repair of a regurgitant, myxomatous valve, and 16 underwent mitral valvuloplasty for prematurely calcified mitral stenosis. The valve was approached with standard instruments through a 5- to 8-cm right parasternal incision. Eighty-five had open femoral artery-femoral vein cannulation, but this technique has recently been replaced by direct cannulation of the aorta and percutaneous cannulation of the femoral vein for most patients. Results: There were no operative deaths. The mean mitral regurgitation score (0-4) decreased from 3.7 to 0.7 after the operation. Although ischemic and bypass times were increased, postoperative recovery was accelerated. Ventilatory support time, intensive care unit stay, hospital stay, need for rehabilitation, and return to "normal activities" all improved. Hospital charges, pain medications, and blood transfusions were also reduced. New atrial fibrillation contributed significantly to increased length of stay and charges. There were no deep wound infections. Other complications included re-exploration for bleeding $(n=1)$, transient ischemic attacks $(n=2)$, stroke $(n=1)$, femoral artery injury (n $=5$ ), pseudoaneurysm $(n=2)$, and antegrade dissection of the ascending aorta $(n=1)$. Two patients died and 1 required reoperation during a mean follow-up of $\mathbf{8 . 8}$ months. Conclusions: Direct-access minimally invasive mitral valve surgery can accelerate recovery, decrease charges, and decrease pain, while maintaining overall surgical efficacy. It has become our standard approach for isolated primary mitral valve operations. ( $J$ Thorac Cardiovasc Surg 1998;116:705-15)
$T^{1}$ he field of minimally invasive cardiac surgery has grown rapidly in recent years. Although diverse techniques and philosophies have emerged, these techniques share the common goal of decreasing surgical trauma while maintaining surgical efficacy. If successful, this reduction in surgical trauma should decrease

From the Division of Cardiac Surgery, Brigham and Women's Hospital, Harvard Medical School, Boston.

Read at the Seventy-eighth Annual Meeting of The American Association for Thoracic Surgery, Boston, Mass, May 3-6, 1998

Received for publication May 8, 1998; revisions requested June 16, 1998; revisions received July 13, 1998; accepted for publication July 16, 1998.

Address for reprints: Lawrence H. Cohn, MD, Division of Cardiac Surgery, Brigham and Women's Hospital, 75 Francis St, Boston, MA 02115.

Copyright $\odot 1998$ by Mosby, Inc.

$0022-5223 / 98 \$ 5.00+0 \quad \mathbf{1 2 / 6 / 9 3 1 1 8}$ pain, reduce morbidity, shorten recovery time, and decrease costs while achieving a superior cosmetic result and improved patient satisfaction. Minimally invasive valve surgery, particularly mitral surgery, has been less widely performed but is increasingly significant. A variety of approaches have been reported, including Port-Access techniques, ${ }^{1,2}$ videoscopic techniques, ${ }^{3-6}$ various types of partial sternotomy, ${ }^{7-12}$ and the parasternal approach. ${ }^{13-17}$

In August 1996 we initiated a minimally invasive direct-access mitral valve surgery program using a parasternal approach originally suggested by Navia and Cosgrove. ${ }^{13}$ Our goal was to develop a system to perform primary mitral valve surgery that fulfilled the following requirements:

1. A significantly smaller incision, with minimal disruption of the chest wall 
Table I. Preoperative patient characteristics

\begin{tabular}{|c|c|c|}
\hline & \multicolumn{2}{|c|}{$\begin{array}{l}\text { Minimally invasive surgery } \\
\qquad(n=106)\end{array}$} \\
\hline & No. & $\%$ \\
\hline \multicolumn{3}{|l|}{ Age (yr) } \\
\hline Mean & $58.1 . \pm 12.7$ & \\
\hline Range & $22-83$ & \\
\hline Male/female & $62 / 44$ & \\
\hline NYHA functional class & $2.3 \pm 0.5$ & \\
\hline \multicolumn{3}{|l|}{ Mitral valve pathophysiology } \\
\hline Mitral regurgitation & 93 & 88 \\
\hline Mitral stenosis & 4 & 4 \\
\hline Mixed & 9 & 8 \\
\hline \multicolumn{3}{|l|}{ Mitral valve pathology } \\
\hline Myxomatous & 83 & 78 \\
\hline Rheumatic & 15 & 14 \\
\hline Endocarditis & 5 & 5 \\
\hline Congenital & 2 & 2 \\
\hline Ischemic & 1 & 1 \\
\hline \multicolumn{3}{|l|}{ Procedure } \\
\hline Mitral valve repair & 90 & 85 \\
\hline Mitral valve replacement & 16 & 15 \\
\hline \multicolumn{3}{|l|}{ Left ventricular function } \\
\hline Mean ejection fraction & $61 \% \pm 12 \%$ & \\
\hline Good $(>50 \%)$ & 93 & 87 \\
\hline Mild dysfunction (40\%-50\%) & 9 & 9 \\
\hline Moderate dysfunction (30\%-40\%) & 3 & 3 \\
\hline Severe dysfunction $(<30 \%)$ & 1 & 1 \\
\hline \multicolumn{3}{|l|}{ Preoperative rhythm } \\
\hline Normal sinus rhythm & 62 & 58 \\
\hline Atrial fibrillation & 43 & 41 \\
\hline Paced & 1 & 1 \\
\hline \multicolumn{3}{|l|}{ Coronary artery disease } \\
\hline None & 91 & 86 \\
\hline One- to two-vessel, no intervention & 6 & 5.5 \\
\hline One- to two-vessel, preop PTCA & 2 & 2 \\
\hline Coronary angiogram not done & 7 & 6.5 \\
\hline
\end{tabular}

NYHA, New York Heart Association; PTCA, percutaneous transluminal coronary angioplasty.

2. A safe and reproducible approach to cardiopulmonary bypass $(\mathrm{CPB})$ and myocardial protection

3. Applicability to the majority of patients with isolated mitral valve disease

4. Adequate visualization to allow standard valve repair and replacement by means of conventional techniques and instruments

5. Equal to the safety and efficacy of conventional mitral valve surgery

In this report we review our techniques and results with this approach to assess the extent to which we have accomplished these goals.

\section{Methods}

Patient population. Between August 1, 1996, and January 1, 1998, 109 patients underwent direct-access minimally invasive mitral valve surgery through a right parasternal incision at Brigham and Women's Hospital. For the purposes of this analysis, the 3 patients who underwent concomitant single coronary artery bypass, subaortic reconstruction, or reoperative surgery were excluded, leaving 106 patients in the study group. Five patients who underwent minor secondary procedures through the right atrium ( 3 atrial septal defect repairs and 3 tricuspid annuloplasties) were included. Three patients had undergone closed mitral commissurotomy $(n=2)$ or balloon valvuloplasty $(n=1)$. Of these 106 patients, 90 underwent mitral valve repair and 16 underwent mitral valve replacement. Preoperative characteristics of the patients are shown in Table I.

Patient characteristics. The majority of patients had severe, chronic mitral regurgitation whereas a smaller number had pure mitral stenosis or a mixed picture of both regurgitation and stenosis. The most common pathologic findings were myxomatous degeneration, rheumatic disease, and endocarditis. Almost all patients had New York Heart Association class II or III symptoms. Left ventricular function was good or only mildly depressed in most patients. The majority were in normal sinus rhythm, but a significant minority had atrial fibrillation before the operation. All patients more than 50 years old underwent coronary angiography; significant multivessel coronary artery disease was a contraindication to the minimally invasive approach. A few patients in each group had mild coronary artery disease and 3 patients had preoperative percutaneous transluminal angioplasty.

\section{Operative techniques}

Preoperative preparation. The patient's body habitus and chest x-ray film were carefully reviewed before a minimally invasive approach was undertaken. Obtaining adequate exposure can be difficult in markedly obese patients, patients with significant chest wall abnormalities (pectus excavatum, deep anteroposterior diameter), and those with unusual cardiac orientation (extremely vertical or horizontal, leftward shift). Transesophageal echocardiography (TEE) was used in all patients and is essential in guiding cannulation, recovering intracardiac air, and in assessing the technical result. A pulmonary artery catheter was inserted in $53 \%$ of patients but in only $20 \%$ over the last 3 months. Its use is now limited to elderly patients, those with decreased left ventricular function, and those with severe pulmonary hypertension. Transcutaneous defibrillator pads were placed on all patients.

Incision. A 5- to 8-cm vertical incision was made along the right edge of the sternum. Assessing the position of the right atrium and ascending aorta relative to the intercostal spaces on the chest x-ray film is critical. Typically, the pericardial space is first entered through a smaller incision directly over the third costal cartilage. This incision is then extended over the second or fourth cartilage depending on the patient's anatomy. Although this approach usually led to resection of the third and fourth cartilages, a large number of patients have had the second and third cartilages resected as we have moved to direct aortic cannulation in recent months.

Dissecting the pectoralis major muscle off its sternal insertion, mobilizing it widely off the chest wall, greatly aids in the exposure. A 1- to 2-cm section of each costal cartilage is 

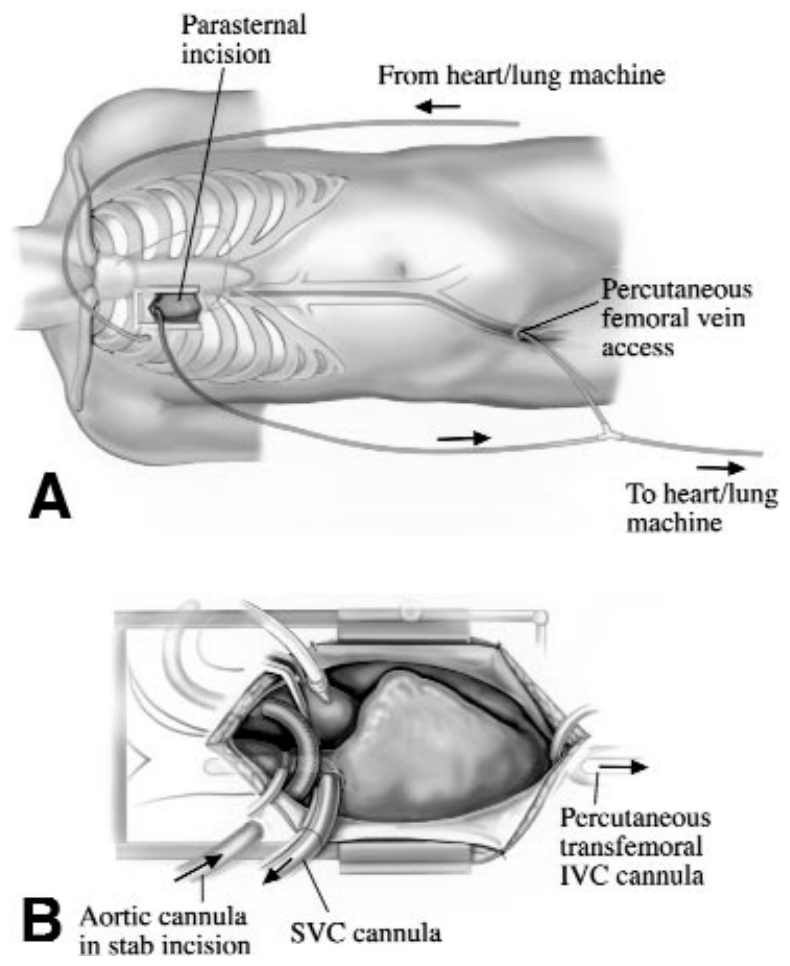

Fig 1. Current cannulation setup for minimally invasive mitral valve surgery. A, Right parasternal incision over third and fourth costal cartilages. Venous drainage through a percutaneous right femoral venous cannula and a superior vena cava cannula. Arterial perfusion through an extended aortic cannula passing through a stab incision into the aorta, positioned in the distal arch. B, Exposure of right atrium after completion of cannulation, placement of aortic crossclamp, initiation of antegrade cardioplegia, and securing of tourniquets around inferior and superior venae cavae (IVC and $S V C$, respectively).

excised at the sternocostal junction. The right internal thoracic pedicle is then divided at the upper and lower intercostal spaces, and the intercostal muscles are incised 3 to 4 $\mathrm{cm}$ lateral to the thoracic pedicle. These maneuvers allow the detached ribs to be retracted laterally like an open book and minimize the amount of cartilage resection necessary to obtain adequate exposure. The right pleural space is entered to prevent postoperative pericardial effusions. The pericardium is incised parallel to the phrenic nerve and marsupialized by securing it to the skin with multiple retraction sutures to pull the heart slightly to the right of midline and into the field.

$C P B$. The majority of patients $(85 \%)$ were supported with CPB by means of femoral cannulation through a small (2 to $4 \mathrm{~cm}$ ) oblique incision above and parallel to the groin crease. The most common cannulas included a 20F cannula (Sarns, Inc, Ann Arbor, Mich) in the right femoral artery, a long 23F to 27F Bio-Medicus cannula (Medtronic Bio-Medicus, Eden Prairie, Minn) inserted into the inferior vena cava through the right femoral vein, and a 24F right-angled cannula (Research

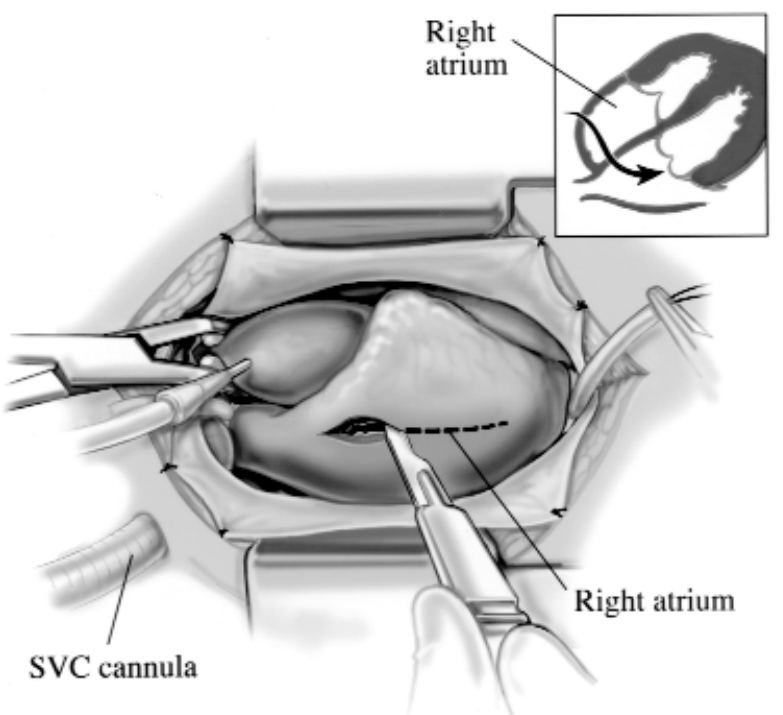

Fig 2. Right atriotomy after completion of cardioplegia. SVC, Superior vena cava.

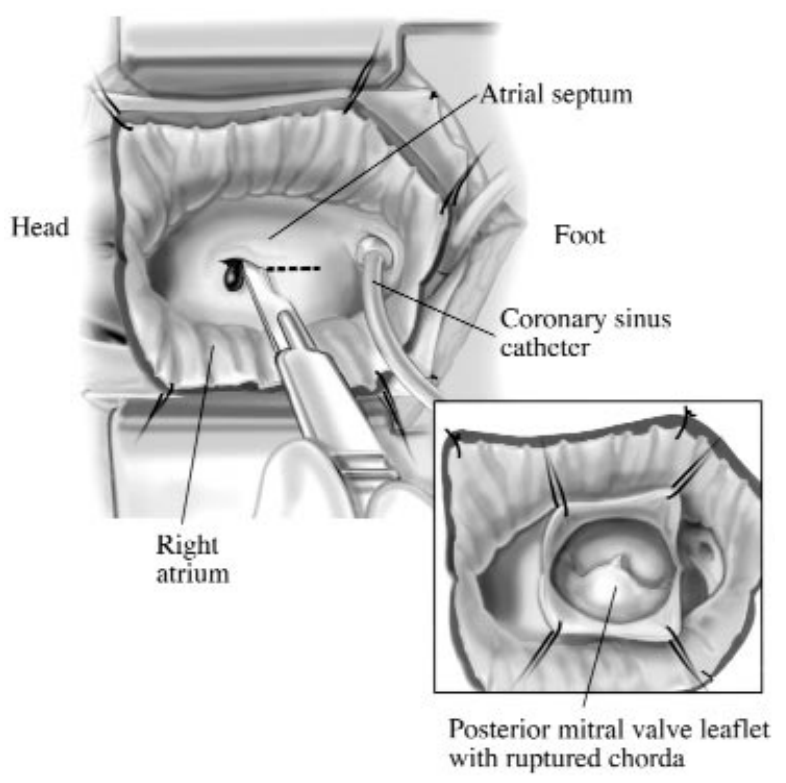

Fig 3. Atrial septotomy after completion of right atriotomy and retraction of cut edges of right atrium with multiple retraction sutures. Optional direct insertion of coronary sinus catheter also shown. Lower panel, Exposure of mitral valve after completion of atrial septotomy and retraction of cut edges of atrial septum with multiple retraction sutures.

Medical, Midvale, Utah) inserted directly into the superior vena cava. The femoral vein cannula was placed through a purse-string suture at the sapheno-femoral junction without clamping the vein.

Two recent developments have allowed us to avoid a groin incision in the majority of patients (11 of the last 16 patients). 


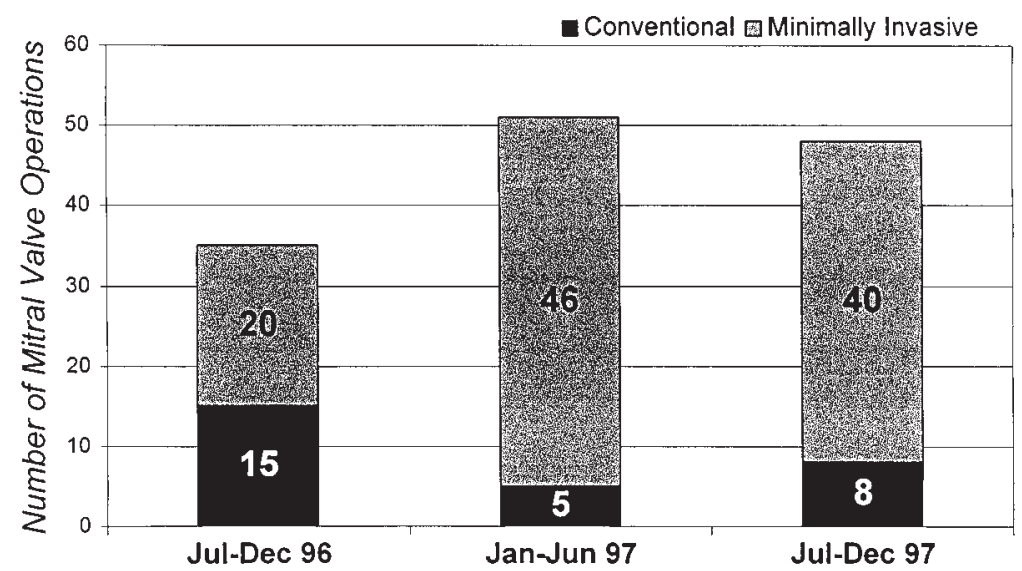

Fig 4. Demonstration of proportion of primary (without coronary artery bypass grafting) mitral valve operations at Brigham and Women's Hospital performed by conventional and minimally invasive techniques (August 1996 to December 1997).

This alternative cannulation technique is depicted in Fig 1. The ascending aorta is cannulated, by means of a modified Seldinger technique, with a $20 \mathrm{~F}$ elongated, thin-walled arterial cannula equipped with a finely tapered but pliable dilator (Medtronic DLP, Grand Rapids, Mich). The wire and cannula tip are positioned in the distal aortic arch by TEE. A $21 \mathrm{~F}$ percutaneous Bio-Medicus cannula is inserted through the femoral vein and guided into the inferior vena cava by TEE. Vacuum-assisted venous drainage has enabled us to use significantly smaller cannulas even in relatively large patients, protecting the exposure by using the smallest possible cannulas and tourniquets and passing them through separate stab incisions (future chest tube sites).

All patients received intermittent antegrade infusions of cold blood cardioplegic solution. Some patients (24\%) received additional retrograde infusions of cardioplegic solution, usually by cannulating the coronary sinus under direct vision. Moderate systemic hypothermia $\left(25^{\circ} \mathrm{C}-30^{\circ} \mathrm{C}\right)$ was generally used to provide an added measure of myocardial protection.

Mitral valve exposure. The mitral valve was exposed through the right atrium and atrial septum in the majority $(76 \%)$ of patients (Figs 2 and 3). Multiple retraction sutures were placed, first on the right atrial incision and then on the septal edges, to gradually obtain exposure of the valve. Placing some or all of the annuloplasty sutures at the outset greatly improves valve exposure by bringing the anulus rightward and into the field. In the remaining patients, generally those with large left atria, the valve was approached directly through the left atrium after dissection of Sondergaard's groove. With either approach, the atrial septum was retracted anteriorly and to the left by means of a blade from the Cosgrove mitral valve retraction system (Baxter Healthcare Corp, Santa Ana, Calif) attached to the articulating arm of a Thompson retractor. Mitral valve repair or replacement was performed with the use of standard instruments and techniques.

Closure. The atrial septotomy and right atriotomy were then closed. A ventricular pacing wire was placed on the right ventricle while the heart was still empty, before the cross- clamp had been removed. Careful complete evacuation of intracardiac air was performed through the left atrium and aortic root and confirmed by TEE. After termination of CPB, the incision was closed by carefully reapproximating the pectoralis major muscle back to the sternum. Paying careful attention to this maneuver and limiting the amount of resected cartilage appear to have eliminated most problems with lung herniation through the parasternal incision. Two small right-angled chest drains were placed through previously placed stab incisions and connected to underwater suction.

Postoperative care. Most patients were extubated in the intensive care unit and transferred to a monitored step-down unit on postoperative day 1 with the goal of discharge by postoperative day 3 to 5 . All patients without contraindications to $\beta$-blockers were started on a program of metoprolol for prophylaxis against atrial fibrillation. Patients with new atrial fibrillation were generally discharged on a regimen of warfarin once adequate rate control had been achieved. All patients underwent transthoracic echocardiography before discharge.

Data collection and analysis. Postoperative follow-up included a 6-week postoperative visit and annual follow-up visits including serial echocardiography when possible. Further follow-up was obtained through telephone calls to every patient during March 1998. Preoperative, operative, and postoperative data were prospectively collected and stored in our division's cardiac surgery database. Hospital charges were adjusted to constant 1998 dollars by means of the annual rate of increase of charges at our hospital.

Data were tabulated with the use of Microsoft Excel and statistical analysis was performed by means of the Prophet statistical package (BBN Systems \& Technologies, Cambridge, Mass). Means are reported along with the standard deviation. Means were compared by use of an unpaired Student $t$ test when the sample passed the normality test. Otherwise, a 2-sample Mann-Whitney rank sum test (2-sided) was used to compare the medians of the samples. Proportions 
Table II. Operative techniques $(n=106)$

\begin{tabular}{lrr}
\hline & No. & $\%$ \\
\hline Incision & & \\
$\quad$ Parasternal & 106 & 100 \\
Conversion to sternotomy & 0 & \\
Mitral valve exposure & & \\
$\quad$ Right atriotomy and atrial septotomy & 80 & 75 \\
$\quad$ Left atriotomy through interatrial groove & 26 & 25 \\
CPB & & \\
Arterial cannulation & 87 & \\
$\quad$ Femoral artery & 19 & 18 \\
$\quad$ Aorta & & \\
$\quad$ Venous cannulation & 5 & 54 \\
$\quad$ Right atrium/SVC & 89 & 11 \\
$\quad$ Femoral vein (open)/SVC & 12 & 76 \\
$\quad$ Femoral vein (closed)/SVC & & 24 \\
Cardioplegia & 81 & \\
Antegrade only & 25 & \\
Antegrade/retrograde &
\end{tabular}

$C P B$, Cardiopulmonary bypass; $S V C$, superior vena cava.

were compared with the use of Fisher's exact test for 2-by-2 contingency tables and $\chi^{2}$ analysis for larger tables.

\section{Results}

During the study period (August 1, 1996, to January 1, 1998), 106 patients underwent minimally invasive mitral valve surgery (MI-MVS group) and 22 patients underwent conventional primary, isolated mitral valve surgery through a full sternotomy (control group). Hence minimally invasive operations represented $83 \%$ of all primary, isolated mitral valve procedures during this period. This proportion increased from $57 \%$ for the second half of 1996 to 87\% during 1997 (Fig 4).

Operative and postoperative data. Specific operative techniques of mitral valve repair and replacement including CPB data are summarized in Tables II and III. Most patients having mitral valve repair underwent posterior leaflet resection with leaflet advancement and ring annuloplasty. The mean ischemic and CPB times were generally about $25 \%$ higher than those needed for conventional mitral valve surgery. Early postoperative data are summarized in Tables IV to VI. Duration of ventilatory support and intensive care unit stay was 8.9 \pm 74 hours and $31 \pm 37$ hours, respectively, for patients having minimally invasive mitral valve surgery (MIMVS group). In a comparison group having conventional mitral valve surgery (control group), nearly twice as many patients required extended ventilatory support of greater than 12 hours and extended intensive care unit stay of greater than 48 hours.

Blood product use. The number of patients requiring no red blood cell transfusions was 67 of $106(63 \%)$ and
Table III. Operative procedures $(n=106)$

\begin{tabular}{|c|c|c|}
\hline & No. & $\%$ \\
\hline Mitral valve repair & 90 & 84 \\
\hline $\begin{array}{l}\text { Posterior leaflet resection } \\
\text { and sliding valvuloplasty }\end{array}$ & 83 & 92 \\
\hline Commissuroplasty & 12 & 13 \\
\hline Chordoplasty & 5 & 6 \\
\hline Anterior leaflet resection & 1 & 1 \\
\hline Commissurotomy & 1 & 1 \\
\hline Cleft closure & 2 & 2 \\
\hline Decalcification & 4 & 4 \\
\hline \multicolumn{3}{|l|}{ Ring annuloplasty* } \\
\hline Cosgrove-Edwards ring & 83 & 92 \\
\hline Carpentier-Edwards ring & 3 & 3 \\
\hline Duran ring & 0 & 0 \\
\hline None & 4 & 4 \\
\hline Mitral valve replacement* & 16 & 15 \\
\hline St Jude Medical & 12 & 75 \\
\hline Hancock & 3 & 19 \\
\hline Carpentier-Edwards & 1 & 6 \\
\hline Secondary procedures & 6 & 6 \\
\hline Atrial septal defect & 3 & 50 \\
\hline Tricuspid annuloplasty & 3 & 50 \\
\hline \multicolumn{3}{|l|}{ Operative times (min) } \\
\hline \multicolumn{3}{|l|}{ Ischemic (crossclamp) } \\
\hline Mean & $102 \pm 35$ & \\
\hline Median & 91.5 & \\
\hline \multicolumn{3}{|l|}{$\mathrm{CPB}$} \\
\hline Mean & $152 \pm 43$ & \\
\hline Median & 142.5 & \\
\hline \multicolumn{3}{|l|}{ Red blood cell transfusion } \\
\hline Mean (units) & $0.9 \pm 2.2$ & \\
\hline None & 67 & 63 \\
\hline$>4$ units & 3 & 3 \\
\hline
\end{tabular}

*Baxter Healthcare Corp, Edwards Division, Santa Ana, Calif; Medtronic, Inc, Minneapolis, Minn; St Jude Medical, Inc, St Paul, Minn.

the mean number of units transfused was $0.9 \pm 22$ units. Only $3(3 \%)$ required more than 4 transfusions (Table III).

Pain control. In-hospital pain control was roughly assessed by tabulating the number of milligrams of morphine and tablets of acetaminophen/oxycodone hydrochloride (Percocet; DuPont Merck Pharmaceutical Company) that were administered within the first 3 postoperative days. The MI-MVS group required $24 \%$ less morphine and $34 \%$ less acetaminophen/oxycodone hydrochloride (Percocet) than other patients undergoing sternotomy in our experience. Pain control after discharge was assessed according to the degree to which the patients took advantage of their Percocet prescription. Fewer than $5 \%$ of patients completed their initial prescription and about $50 \%$ of patients in the MI-MVS group reported that they never even filled the initial pain medicine prescription.

Hospital charges. Mean hospital charges, expressed 
Table IV. Operative mortality and morbidity $(n=106)$

\begin{tabular}{lcc}
\hline & No. & $\%$ \\
\hline Operative deaths & 0 & 0 \\
Cardiac morbidity & 17 & 27 \\
New atrial fibrillation & 16 & 94 \\
$\quad$ Reverted to NSR by 6 wk & 1 & 0.9 \\
New permanent pacemaker & 1 & 0.9 \\
Inotropic support & 1 & 0.9 \\
IABP & 0 & 0.0 \\
Myocardial infarction & 3 & 2.8 \\
Pericardial effusion necessitating drainage & & \\
Other morbidity & 1 & 0.9 \\
Re-exploration for bleeding & & \\
Neurologic events & 2 & 1.9 \\
$\quad$ Transient ischemic attack & 1 & 0.9 \\
$\quad$ Stroke & & \\
Wound problems & & 0 \\
$\quad$ Chest & 0 & 0.9 \\
$\quad$ Deep infection & 3 & 0.9 \\
$\quad$ Superficial infection & 1 & \\
$\quad$ Herniation (troublesome) & & 0.9 \\
Groin & 1 & 3.7 \\
$\quad$ Major (lymphocele) & & \\
$\quad$ Minor & 4 & 0.9 \\
Vascular events & & \\
Antegrade ascending aortic dissection & 1 & 5 \\
$\quad$ Femoral artery problems & 2 & \\
Pseudoaneurysm & 1 & \\
Femoral artery embolism & & \\
\hline
\end{tabular}

NSR, Normal sinus rhythm; IABP, intra-aortic balloon pump.

in constant 1998 dollars, were about $\$ 41,000.00$. New atrial fibrillation had a major impact on hospital charges, as it did on length of stay, increasing charges by $\$ 8843$.

Early morbidity and mortality. Early postoperative morbidity and mortality for the MI-MVS group are shown in Table IV.

Operative mortality. There were no operative deaths in the MI-MVS group.

Cardiac morbidity. Low cardiac output necessitating moderate inotropic support (dopamine $>5 \mu \mathrm{g} / \mathrm{kg}$ per minute) occurred in only 1 patient. No myocardial infarctions developed. One patient, without significant coronary artery disease, required placement of an intraaortic balloon pump for severe, persistent ischemic changes after being weaned from CPB. These changes resolved completely within 24 hours, without definite chemical, electrocardiographic, or echocardiographic evidence of myocardial infarction. New postoperative atrial fibrillation occurred in $27 \%$ of those patients who had normal sinus rhythm on admission. In only 1 of these patients had normal sinus rhythm not been restored by the 6-week postoperative visit. One patient required placement of a permanent pacemaker for post-
Table V. Postoperative data $(n=106)$

\begin{tabular}{|c|c|c|}
\hline \multicolumn{3}{|l|}{ Recovery times } \\
\hline \multicolumn{3}{|l|}{ Ventilatory support } \\
\hline Mean (h) & $8.9 \pm 7.4$ & \\
\hline Median (h) & 7.5 & \\
\hline$>12 \mathrm{~h}$ (patients) & 23 & $(22 \%)$ \\
\hline \multicolumn{3}{|l|}{ Intensive care unit stay } \\
\hline Mean (h) & $31 \pm 37$ & \\
\hline Median (h) & 23 & \\
\hline$>48 \mathrm{~h}$ (patients) & 9 & $(8 \%)$ \\
\hline \multicolumn{3}{|l|}{ Hospital stay } \\
\hline Mean (d) & $5.6 \pm 3.0$ & \\
\hline$<5 \mathrm{~d}$ (patients) & 40 & $(38 \%)$ \\
\hline$>7$ d (patients) & 14 & $(13 \%)$ \\
\hline Transfer to rehabilitation facility (patients) & 7 & $(7 \%)$ \\
\hline \multicolumn{3}{|l|}{ Return to "normal" activity } \\
\hline Mean (wk) & $9.0 \pm 8.5$ & \\
\hline Median (wk) & 6 & \\
\hline \multicolumn{3}{|l|}{ Pain control } \\
\hline \multicolumn{3}{|l|}{ Morphine intake* } \\
\hline Mean (mg) & $7.3 \pm 5.8$ & \\
\hline Median (mg) & 6 & \\
\hline \multicolumn{3}{|l|}{ In-hospital pain medicine intake* } \\
\hline Mean (tablets) & $6.5 \pm 5.2$ & \\
\hline Median (tablets) & 6 & \\
\hline \multicolumn{3}{|l|}{ Post-hospital pain medicine (patients) } \\
\hline Never filled & 43 & $(49 \%)$ \\
\hline Filled but did not complete & 40 & $(46 \%)$ \\
\hline Completed & 4 & $(5 \%)$ \\
\hline
\end{tabular}

*Total intake for the first 3 postoperative days.

operative complete heart block. Three patients had pericardial effusions that required operative $(n=2)$ or percutaneous drainage $(\mathrm{n}=1)$.

Wound problems. No patient required conversion to sternotomy and none had a deep sternal infection. Three had superficial chest wound infections: two responded to outpatient antibiotic therapy and 1 required readmission for intravenous antibiotics. Nineteen patients reported mild bulging at their parasternal incision with coughing. Most of these patients were relatively thin, small women, relatively early in our experience. Only 1 of these patients reported this to be a significant problem; the rest remained very satisfied with their choice of a minimally invasive incision. None has required operative intervention.

Four patients had minor groin wound problems including superficial wound infections, seromas, and small hematomas, all of which resolved with conservative treatment. One patient had a lymphocele that ultimately required surgical repair. One patient had femoral neuropathy, which resolved completely.

Vascular problems. Six patients (5.7\%) had vascular problems related to femoral cannulation. Five patients had localized femoral artery injury that was repaired with endarterectomy, primary repair, or Dacron patch 
Table VI. Impact of AF on hospital length of stay

\begin{tabular}{lc}
\hline & Mean length of stay $(d)$ \\
\hline All patients & $5.6 \pm 3.0$ \\
Patients with preop NSR & $5.1 \pm 1.8$ \\
Patients continuing to have NSR & $4.5 \pm 0.9$ \\
Patients with new AF & $6.6 \pm 2.4$ \\
Patients with preop AF & $6.4 \pm 4.1$
\end{tabular}

$A F$, Atrial fibrillation; $N S R$, Normal sinus rhythm.

angioplasty in the operating room. One of these patients had postoperative ischemia in this leg, necessitating operative thrombectomy, and another required operative repair of a pseudoaneurysm. One other patient had a small pseudoaneurysm that was successfully treated with ultrasound-guided compression. All of the remaining patients had palpable distal pulses at discharge and no further sequelae. One patient had an acute thromboembolic event to the noncannulated leg, which necessitated operative thrombectomy. One patient had an antegrade dissection of the ascending aorta in the operating room arising from the cardioplegia needle hole. The ascending aorta was replaced with a tube graft by way of the same incision with the patient under deep hypothermic circulatory arrest. He recovered with no further complications.

Other morbidity. One patient required re-exploration for bleeding from a branch of the right internal thoracic artery, 1 had a temporary visual ischemic attack, and 2 had small strokes.

Length of stay. The mean length of stay in the hospital was $5.6 \pm 3$ days versus $8.3 \pm 6$ for conventional surgery. The primary reasons for prolonged length of stay in the MI-MVS group appeared to be atrial fibrillation (Table V). None of the 47 patients who remained in normal sinus rhythm throughout their hospitalization had a prolonged length of stay, and the mean length of stay among these patients was 4.5 days, more than 2 days shorter than that of patients who had new atrial fibrillation. Only 7 of 106 patients required post-hospital rehabilitation, and 5 of these 7 were more than 75 years old. On average, patients were able to return to their "normal" activities in half the time required by patients with conventional mitral valve operations performed through a sternotomy.

Surgical efficacy and late results. Surgical efficacy was assessed by reviewing preoperative and postoperative echocardiographic data and functional class. This information is compiled in Table VII along with late postoperative events.

Follow-up. Patient follow-up was 93\% complete. Mean follow-up time was 9 months.

Echocardiographic results. Among those patients undergoing mitral valve repair for mitral regurgitation,
Table VII. Surgical efficacy and late results

\begin{tabular}{lcc}
\hline & No. & $\%$ \\
\hline Mean follow-up time (mo) & $8.9 \pm 3.3$ & \\
$\quad$ Late deaths & 2 & 1.9 \\
$\quad$ Sudden death & 1 & \\
$\quad$ Pneumonia & 1 & 0.9 \\
Reoperations & & \\
Thromboembolic events & 1 & 0.9 \\
$\quad$ Transient ischemic attack & 1 & \\
$\quad$ Stroke & & \\
Echocardiographic results & & \\
$\quad$ Mitral valve repair & $3.8 \pm 0.4$ & \\
$\quad$ Preop MR (0-4) & $0.7 \pm 0.7$ & \\
$\quad$ Postop MR (0-4) & & \\
$\quad$ Mitral valve replacement & 0 & \\
$\quad$ Perivalvular leak & & \\
Functional class (I-IV) & & \\
Preop & & \\
Late postop & & \\
\hline
\end{tabular}

$M R$, Mitral regurgitation.

the mean score (0-4 scale) decreased from 3.7 to 0.7 . No patients undergoing mitral valve replacement were found to have a perivalvular leak.

Late mortality. Two patients died during the followup period. One patient died suddenly 8.5 months after the operation. The other patient had multiple complications during her hospital stay, including a stroke that left her incapacitated, and she died of pneumonia at a rehabilitation facility 7 weeks after the operation.

Late events. One patient underwent reoperative mitral valve replacement 3 months after the operation for recurrent mitral regurgitation and hemolysis. This was due to rupture of a second chorda. One visual transient ischemic attack occurred, but no frank strokes were noted during late follow-up.

\section{Discussion}

The current interest in minimally invasive cardiac surgery has been motivated by a desire to improve patient outcomes by decreasing surgical trauma while maintaining the quality of conventional cardiac surgery. The precise definition of a "minimally invasive" approach and the best way to assess it and other risks and benefits continue to evolve. The encouraging results described herein do seem to indicate a significant benefit to this approach. Nonetheless, several potential issues remain to be addressed.

Patient population. The 106 patients in the study group represent a fairly typical spectrum of adult patients with mitral valve disease in the Western world. They were a relatively young, generally healthy group of patients with good left ventricular function who typically underwent elective mitral valve repair of a myx- 
omatous, regurgitant valve after having class II or III symptoms. Although not a consecutive series, they represent the vast majority of patients undergoing isolated, primary mitral valve surgery at our institution during this time.

Potential sources of bias include the possibility that the higher-risk patients were undergoing conventional operations during this time and that the increased volume of surgery may have generated a referral bias by attracting more low-risk patients. The 22 patients who underwent conventional operations during this period did so primarily as a result of surgeon preference. Therefore, although it is difficult to characterize and compare such a small group of patients, our impression is that these patients were not a higher-risk group than the MI-MVS group.

Technical considerations. The goal of our current approach is to provide, through a limited incision, adequate exposure of the valve to ultimately permit the use of standard techniques of mitral valve repair and replacement. Given the outstanding results of standard mitral valve operations, especially valve repair procedures, ${ }^{18-20}$ it seems difficult to justify compromising the repair for the sake of a smaller incision.

Physical examination and good posteroanterior and lateral chest $\mathrm{x}$-ray films sufficed, in our experience. A very obese or very muscular chest wall is not a good setting for this approach. Obtaining exposure requires a gradual, step-wise approach with liberal use of retraction sutures to rotate the valve into the field. Dividing the right thoracic artery has been very useful in obtaining exposure while minimizing the extent of cartilage resection. Although division of this artery is not ideal, none of these patients had coronary disease and they are unlikely to need this conduit in the future. This technique allows the detached ribs to be retracted laterally without avulsing the artery. Marsupializing the pericardium to the dermis before inserting the retractor has also been very helpful, especially in heavy patients. Protecting the cross-sectional area of the primary wound by passing cannulas and tourniquets through separate stab incisions is helpful. Although each individual item may not seem to be impeding the view, a few items can quickly take up much of the working area of the wound.

In a few patients in whom the third and fourth cartilages appeared to provide adequate exposure, but in whom we encountered difficulties with femoral cannulation, we extended the incision into the second cartilage to allow direct aortic cannulation. If the above guidelines are followed, adequate exposure can be obtained without violating 3 cartilages. Despite these ing phase, no patient required conversion to sternotomy because of dissection at the cardioplegia port.

The 6 patients $(6 \%)$ who had femoral artery complications in this series confirm that groin cannulation has risks. These injuries were all amenable to local repair during the mitral valve operation. Only 2 of these patients, however, required operative reintervention (1 pseudoaneurysm repair and 1 thrombectomy). Whether these localized injuries will result in late-term complications is not known. However, the ability to avoid a groin incision altogether in most patients has been a major advance in this technique. Advanced cannula technology has permitted safe and effective direct aortic cannulation and percutaneous femoral venous cannulation.

Early results. The operative mortality of $0 \%$ and the excellent early echocardiographic results certainly attest to the safety and efficacy of this approach, similar to that of conventional mitral valve surgery. ${ }^{18-20}$ However, the minimally invasive technique clearly required more time in the operating room. The net increase in operating room time was about 65 minutes and can be mostly accounted for by an extra 20 minutes of ischemic time and an extra 45 minutes of CPB. The extra ischemic time is the result of some additional technical maneuvers to enhance exposure. The extra CPB time is mostly due to extra precautions necessary to properly remove air from the heart by echocardiographic control.

Despite this increase in operative times, procedures appear to accelerate all components of inpatient and outpatient recovery, and we believe that this improvement can be attributed to a cumulative effect of less surgical trauma. Significantly shorter times for ventilatory support, intensive care unit stay, hospital stay, and postdischarge return to normal activities were noted. The rate of re-exploration for bleeding was gratifyingly low at $0.9 \%$ (1/106). This, as well as the decreased transfusion requirement, again probably directly reflects the benefits of decreasing surgical trauma and dissection.

Tabulating the consumption of pain medication is a relatively crude measure of pain control compared with other more sophisticated techniques, which we have not yet incorporated into our study. Nonetheless, the significant decrease in inpatient pain medication use, the fact that more than $50 \%$ of patients took no narcotic pain medications after discharge, and the fact that fewer than $5 \%$ finished their prescriptions are all consistent with our impression that these patients have less pain during their early postdischarge recovery. ${ }^{21}$

Although the incidence of new atrial fibrillation was somewhat decreased in the MI-MVS group, it remains a formidable problem that clearly limits our ability to 
shorten length of stay and decrease hospital costs with this procedure, as previously documented. ${ }^{22}$ This is demonstrated by the fact that all of the 47 patients who remained in normal sinus rhythm were discharged by the sixth postoperative day and that new atrial fibrillation added 2.1 hospital days and nearly $\$ 9000$ in extra hospital charges.

Other minimally invasive approaches. Other minimally invasive approaches include video-assisted techniques, Port-Access approaches, and direct-access parasternal or ministernotomy incisions. Many of these reports have been limited to a few patients and have concentrated on technical issues. However, a few groups have collected large enough series to permit analysis of the relative risks and benefits of each technique.

The first "minimally invasive" mitral repair has been attributed to Carpentier's group. ${ }^{3}$ They recently reported on 22 patients in whom video-assisted mitral valve surgery was performed through a 6- to 7-cm minithoracotomy or ministernotomy with the use of femoral cannulation and video assistance. They reported 1 conversion to sternotomy and 3 early reoperations -2 for bleeding and 1 for recurrent mitral regurgitation. They ${ }^{4}$ have recently favored the hemisternotomy with video assistance and have moved, as we have, toward direct aortic cannulation combined with percutaneous femoral venous cannulation.

Chitwood's group ${ }^{5}$ has also advocated video-assisted mitral surgery through a 6-cm anterior thoracotomy using femoral cannulation and a new transthoracic aortic clamp. In a series of 31 patients, they reported 1 operative death and some results that are similar to ours, including higher operating room times followed by accelerated postoperative recovery and decreased costs when compared with historical controls.

The New York University group recently presented encouraging results with Port-Access mitral valve surgery using the Heartport system (Heartport, Inc, Redwood City, Calif) with a $1.1 \%$ mortality and accelerated recovery in 130 patients. ${ }^{1}$ Mohr's group ${ }^{2}$ from Leipzig recently presented data on Port-Access mitral valve surgery in 51 patients with an operative mortality of $10 \%$, with 2 retrograde aortic dissections and 3 early perivalvular leaks.

Finally, Navia and Cosgrove ${ }^{13}$ and Cosgrove, Sabik, and Navia ${ }^{14}$ were the first to popularize the parasternal approach for the mitral valve and described 25 patients in their limited report with techniques very similar to the ones we have used in this series. Some differences include the length of the incision $(10 \mathrm{~cm}$ vs $6-8 \mathrm{~cm})$, complete resection of the costal cartilages (vs partial resection with lateral mobilization through the inter- costal spaces), and a more extensive atriotomy. Cosgrove, Sabik, and Navia ${ }^{14}$ have recently changed their approach to an upper sternotomy for mitral valve surgery to "optimize exposure."

\section{Conclusions}

By eliminating open femoral cannulation in most patients and modifying the specific techniques of the parasternal incision to avoid herniation and paradoxic motion, the direct-access parasternal technique described in this report might serve as a paradigm for the future of minimally invasive mitral valve surgery. Although future technologic and surgical advances will continue to refine the technique, it currently appears to strike a balance between minimizing surgical trauma, by leaving the sternum completely intact, and providing enough exposure to perform a meticulous mitral valve operation without technical compromise. Our results show that although this may add time in the operating room, the patient ultimately benefits by recovering more rapidly, leaving the hospital sooner, with a better cosmetic result, less pain, and less exposure to blood. The recuperation period is significantly shorter and return to normal activities is quicker. Most important, these benefits were accomplished while maintaining or surpassing the surgical efficacy of conventional mitral valve surgery.

\section{REFERENCES}

1. Spencer FC, Galloway AC, Grossi EA, et al. Recent developments and evolving techniques in mitral valve reconstruction. Ann Thorac Surg 1998;65:307-13.

2. Mohr FW, Falk V, Diegeler A, et al. Minimally invasive PortAccess mitral valve surgery. J Thorac Cardiovasc Surg 1998; 115:567-76.

3. Carpentier A, Loulmet D, Carpentier A, et al. Chirurgie a coeur par video-chirurgie et mini-thoracotomie: premier cas (valvuloplastie mitrale) opere succes [first open heart operation (mitral valvuloplasty) under videosurgery through a minithoracotomy]. Comptes Rendus De L'Academie des Sciences: Sciences de la Vie 1996;319:219-23.

4. Loulmet DF, Carpentier A, Cho PW, et al. Less invasive techniques for mitral valve surgery. J Thorac Cardiovasc Surg 1998;115:772-9.

5. Chitwood WR, Elbeery JR, Chapman WHH, et al. Video-assisted minimally invasive mitral valve surgery: the "micro-mitral" operation. J Thorac Cardiovasc Surgery 1997;113:413-4.

6. Lin PJ, Chang $\mathrm{CH}$, Chu JJ, et al. Video-assisted mitral valve operations. Ann Thorac Surg 1996;61:1781-7.

7. Gundry SR, Shattuck OH, Razzouk AJ, et al. Fasile minimally invasive cardiac surgery via mini-sternotomy. Ann Thorac Surg 1998;65:1100-4.

8. Konertz W, Waldenberger F, Schmutzler M, Rittler J, Liu J. Minimal access valve surgery through superior partial sternotomy: a preliminary study. J Heart Valve Dis 1996;5:638-40. 
9. Svensson LG. Minimal-access "J" sternotomy for valvular, aortic and coronary operations or reoperations. Ann Thorac Surg 1997;64:1501-3.

10. Tam RKW, Ho C, Almeida AA. Minimally invasive mitral valve surgery. J Thorac Cardiovasc 1998;115:246-7.

11. Doty DB, DiRusso GB, Doty JR. Full-spectrum cardiac surgery through a minimal incision: mini-sternotomy (lower half) technique. Ann Thorac Surg 1998;65:573-7.

12. Kasegawa H, Shimokawa T, Matsushita Y, Kamata S, Ida T, Kawase M. Right-sided partial sternotomy for minimally invasive operation: "open door method." Ann Thorac Surg 1998;65:569-70.

13. Navia JL, Cosgrove DM. Minimally invasive mitral valve operations. Ann Thorac Surg 1996;62:1542-4.

14. Cosgrove DM, Sabik JF, Navia JL. Minimally invasive valve operation. Ann Thorac Surg 1998;65:1535-9.

15. Cohn LH, Adams DH, Couper GS, et al. Minimally invasive cardiac valve surgery improves patient satisfaction while reducing costs of cardiac valve replacements and repair. Ann Surg 1997;226:421-6.

16. Lazzara RR, Kidwell FE. Right parasternal incision: a uniform minimally invasive A approach for valve operations. Ann Thorac Surg 1998;65:271-2.

17. Cohn LH. Mitral valve repair. Operative techniques in thoracic and cardiovascular surgery. Philadelphia: WB Saunders; 1998; 3:109-25.

18. Cohn LH, Couper GS, Aranki SF, et al. Long-term results of mitral valve reconstruction for regurgitation of the myxomatous mitral valve. J Thorac Cardiovasc Surg 1994;107:143-51.

19. Cosgrove DM, Aricidi JM, Rodriguez L, et al. Initial experience with the Cosgrove-Edwards annuloplasty system. Ann Thorac Surg 1995;60:499-504.

20. Carpentier A. Cardiac valve surgery-the "French correction." J Thorac Cardiovasc Surg 1983;86:323-47.

21. Cohn LH, Adams DH, Couper GS, et al. Minimally invasive cardiac surgery improves patient satisfaction while reducing costs of cardiac valve replacement and repair. Ann Surg 1997;226:421-8.

22. Aranki SF, Shaw DP, Adams DH, et al. Predictors of atrial fibrillation following coronary artery surgery: current trends and impacts on hospital residence. Circulation 1996;94:390-7.

\section{Discussion}

Dr Alain F. Carpentier (Paris, France). This paper is a significant contribution to minimally invasive mitral valve surgery. The Brigham and Women's Hospital group should be congratulated for an innovative technique and for their remarkable results. In particular, they have minimized the parasternal approach and avoided an extensive incision of the 2 atria by approaching the mitral valve through the septum, as we do. However, the parasternal approach is still too invasive. In our institution we have been using the partial sternotomy and transseptal approach with similarly good results, without the risk of lung hernia, the main disadvantage of dividing the internal thoracic artery. Have you tried any of the partial median sternotomy approaches with video assistance, and what is your opinion of them?

Second, I agree with you that aortic cannulation should be preferred to femoral cannulation. Of course, the cannula obscures somewhat the operative field, but in compensation, you can avoid cannulating the superior vena cava by using the double-staged venous cannula developed by DLP, Inc (Walker, Mich), which provides an excellent drainage of the 2 venae cavae through this single cannula introduced through the ileac vein up to the superior vena cava.

Third, you have obtained excellent short-term results with no mortality and a low degree of residual mitral regurgitation. Rather than telling us the residual regurgitation score, it would be more informative to tell us how many cases of 0 , $1+$, or $2+$ residual regurgitation you observed.

I raise this important question because one of the risks of minimally invasive mitral valve surgery is to perform a less optimal repair, favoring, for example, a less durable posterior support of the posterior leaflet rather than a complete remodeling of the anulus whenever necessary. I do not recall any data comparing residual insufficiency in patients having minimally invasive operations and those undergoing conventional techniques.

Another risk of minimally invasive mitral valve surgery is the temptation to favor valve replacement instead of valve repair. I noticed that in the period between January and June 1997, you had a proportion of approximately $10 \%$ valve replacements versus $90 \%$ valve repairs, which is very good, but during the following 6-month period the proportion of valve replacements rose to approximately $17 \%$. Does that mean that you experienced some disenchantment?

Your data comparing minimally invasive surgery to conventional surgery in terms of postoperative recovery, ventilatory support time, and intensive care unit and hospital stay were impressive; however, there may be a bias in this regard since the fast-track policy began at approximately the same time as minimally invasive surgery. Do you think that the difference you showed would be less impressive today with the fast-track conventional surgery used in most institutions?

Finally, I agree with you that minimally invasive surgery is feasible with excellent results. However, minimally invasive mitral valve surgery is more difficult than aortic valve surgery and carries on a risk of suboptimal valve repair, which has not been the case in your study.

Dr Aklog. Thank you, Dr Carpentier. We did sacrifice the right internal thoracic artery in all of these patients. The risk of infection has not been a problem. More important, because these patients all have normal coronary arteries, it is extremely unlikely that they will need the internal thoracic artery as a potential conduit in the future. We believe that dividing the right internal thoracic artery does help with mobilization by allowing greater retraction of the lateral aspect of the wound while minimizing the amount of resected cartilage.

We have not tried a partial sternotomy approach for mitral valve surgery. However, we have used this approach extensively for aortic valve surgery.

You commented on the double-staged venous cannula, which avoids the need for a superior vena cava cannula. I have seen that in your report. I think it will be a helpful contribution to this approach. Any item that can be removed from the main wound does improve exposure and consequently improves the ease of the operation. 
You asked about the relative proportion of patients who had different degrees of residual mitral regurgitation after repair. Two patients had 3+/4+ mitral regurgitation. One of these patients underwent reoperation, as described. Ten patients had $2+/ 4+$ mitral regurgitation, and the rest of them had 0 or $1+/ 4$. The patients having minimally invasive operations actually had a slightly lower score, but the difference was not statistically significant. Therefore one major point that we wanted to convey was that this exposure provides an excellent view of the valve and allows the operation to be done in the conventional way with standard instruments. The limited exposure did not compromise the repair in any way. Our data indicate that we were able to maintain the efficacy of the conventional mitral valve repair.

We did not favor replacement over repair in the minimally invasive group. I think the figure you are referring to is the one comparing the rate of minimally invasive versus conventional surgery. The proportion of repairs has remained about the same during the minimally invasive period and was, in fact, significantly higher than in the conventional group.

Our fast-track program was in place before treatment of the control group was begun. Clearly this is a historical control group and contains the usual limitations of such a group. However, I do not think the differences in fast-track policy were a major factor in explaining our encouraging results. Although even after the initiation of our fast-track program, there has been ongoing pressure to get patients out of the hospital, we do not think this can account for the significant decrease in length of stay seen in these patients. We, in fact, evaluated whether there were comparable decreases in stay for other operations, such as routine coronary procedures, and found that the magnitude of the decrease was small compared with what we saw with the initiation of minimally invasive mitral valve surgery. Again, within the limitations of a historical control group, we think that the patients undergoing minimally invasive operations did recover more rapidly.

Dr David B. Skinner (New York, NY). In view of the importance of bilateral internal thoracic artery grafting, is it necessary to divide the right internal thoracic artery?

Second, your abstract listed 100 patients and your article, 106. What is the reason for this discrepancy?

Dr Aklog. We added 6 additional patients from the date of the abstract to December 31, 1997.

We have, over time, decreased the amount of cartilage resected to decrease the potential problem with wound herniation. One of our techniques to accomplish this is to incise through the intercostal space above and below, quite laterally. This allows the incision to open up laterally like an open book. To accomplish that without the potential of avulsing the thoracic artery during retraction, we have to divide the artery at the 2 intercostal spaces. As I said, these patients have no coronary disease. If we were sacrificing the left internal thoracic artery, we would be more concerned. However, with the right, we have taken the gamble that these patients are not going to need it as a conduit in the future.

\section{Bound volumes available to subscribers}

Bound volumes of The Journal of Thoracic and Cardiovascular Surgery are available to subscribers (only) for the 1998 issues from the Publisher, at a cost of \$122.00 for domestic, \$151.94 for Canadian, and \$142.00 for international subscribers for Vol 115 (January-June) and Vol 116 (July-December). Shipping charges are included. Each bound volume contains a subject and author index and all advertising is removed. Copies are shipped within 60 days after publication of the last issue of the volume. The binding is durable buckram with the Journal name, volume number, and year stamped in gold on the spine. Payment must accompany all orders. Contact Mosby, Inc, Subscription Services, 11830 Westline Industrial Drive, St Louis, MO 63146-3318, USA; phone 800-453-4351 or 314-453-4351.

Subscriptions must be in force to qualify. Bound volumes are not available in place of a regular Journal subscription. 